\title{
Low expression of RecQ-like helicase 5 is associated with poor prognosis in patients with gastric cancer
}

\author{
YIJIA LIN $^{1 *}$, HUASHE WANG $^{1 *}$, XINYOU WANG ${ }^{1}$, MIAO LI $^{2}$, HONGLEI CHEN $^{3}$ and JUNSHENG PENG ${ }^{1}$ \\ Departments of ${ }^{1}$ Gastrointestinal Surgery and ${ }^{2}$ Digestion, Guangdong Provincial Key Laboratory of Colorectal and Pelvic \\ Floor Diseases, The Sixth Affiliated Hospital, Sun Yat-sen University, Guangzhou, Guangdong 510655; \\ ${ }^{3}$ Gastrointestinal Endoscopy Center, The Eighth Affiliated Hospital, Sun Yat-sen University, \\ Shenzhen, Guangdong 518033, P.R. China
}

Received June 9, 2019; Accepted October 25, 2019

DOI: $10.3892 / \mathrm{ol} .2019 .11137$

\begin{abstract}
The role of RecQ-like helicase 5 (RECQL5) in gastric cancer (GC) is unclear. This study investigated the expression, clinicopathological association and prognosis of RECQL5 protein in human GC. Firstly, the Oncomine database was used to determine the mRNA expression levels of RECQL5 in GC samples. GC samples and adjacent normal gastric tissue samples were subsequently assessed to determine RECQL5 protein expression levels using immunohistochemistry. The clinicopathological association with RECQL5 expression was analyzed. Multivariate Cox analysis was performed to determine the relationship between RECQL5 expression and survival outcomes. Data from the Oncomine database revealed that RECQL5 mRNA was significantly downregulated in GC tissues compared with that in normal gastric tissues $(\mathrm{P}<0.05)$. These results were then validated at the protein level as RECQL5 protein expression was found to be significantly downregulated in GC samples compared with that in normal gastric tissues $(\mathrm{P}<0.05)$. Low expression of RECQL5 was significantly associated with depth of tumor invasion, histological differentiation and TNM stage $($ all $\mathrm{P}<0.05)$ and indicated poor prognosis in patients with GC. Multivariate analysis revealed that low
\end{abstract}

Correspondence to: Professor Junsheng Peng, Department of Gastrointestinal Surgery, Guangdong Provincial Key Laboratory of Colorectal and Pelvic Floor Diseases, The Sixth Affiliated Hospital, Sun Yat-sen University, 26 Yuancun Er Heng Road, Tianhe, Guangzhou, Guangdong 510655, P.R. China

E-mail: pengsir1010@126.com

Professor Honglei Chen, Gastrointestinal Endoscopy Center, The Eighth Affiliated Hospital, Sun Yat-sen University, No. 3025 Shennan Zhong Road, Futian, Shenzhen, Guangdong 518033, P.R. China

E-mail: chl_830830@163.com

*Contributed equally

Key words: RecQ-like helicase 5, gastric cancer, immunohistochemistry, prognosis
RECQL5 expression and depth of invasion were independent prognostic factors for $\mathrm{GC}(\mathrm{P}<0.05)$. These results suggest that low expression of RECQL5 is associated with carcinogenesis and invasion in GC and with poor overall survival in patients with GC. RECQL5 may be a novel prognostic marker for patients with GC.

\section{Introduction}

Gastric cancer (GC) has been considered as a common primary malignant tumor with the fourth-highest cancer-related mortality rate worldwide in the last decade (1). In China in $2015, \geq 60 \%$ of patients with GC had advanced disease (2). In the last decade, with the development of GC therapy, many cancer markers such as programmed death-ligand 1 and human epidermal growth factor receptor 2 have been assessed as candidate prognostic factors and therapeutic targets for GC $(3,4)$. The 5-year survival rate and quality of life of patients with GC has improved markedly (5-7). However, patients in the advanced stage still have a poor prognosis, and advanced GC poses a higher burden for patients and society (8). Thus, markers that accurately predict the prognosis of patients with GC are needed.

RecQ helicases play a critical role in maintaining genome stability, as well as DNA recombination, replication and transcription (9-11). There are five RecQ helicases in human cells: RecQ-like helicase 1 (RECQL1), Werner syndrome RecQ-like helicase (WRN), Bloom syndrome RecQ-like helicase (BLM), RecQ-like helicase 4 (RECQL4) and RecQ-like helicase 5 (RECQL5) $(9,10,12)$ Mutations in WRN, BLM and RECQL4 proteins can lead to genomic instability and predisposition to cancers, including colorectal, prostate and breast cancers (13-16). Important roles of RECQL5 have been identified in DNA replication and transcription, base excision repair and homologous recombination $(17,18)$. Lao et al (19) reported the abrogation of RECQL5 expression in colorectal cancer. Another study demonstrated that RECQL5 acts as a tumor suppressor in osteosarcoma, and increased expression of RECQL5 can inhibit the progression of osteosarcoma (20). Conversely, other studies showed that RECQL5 is overexpressed in breast cancer and bladder carcinoma, and that depletion of RECQL5 can significantly reduce the progression of cancer $(21,22)$. However, the roles of RECQL5 in GC remains unclear. 
In the present study, expression of RECQL5 was investigated by mining the publicly available Oncomine database, combined with validation in samples from patients with GC and normal adjacent tissues using immunohistochemistry. The clinicopathological and prognostic significance of RECQL5 in patients with GC was also evaluated.

\section{Materials and methods}

Bioinformatics prediction. The RECQL5 mRNA data from GC and normal gastric tissues were extracted from the Oncomine online database (https://www.oncomine.org). The filtered datasets were analyzed separately. RECQL5 expression values between normal gastric tissues and GC tissues were extracted and compared from the Chen Gastric, DErrico Gastric and Cho Gastric datasets (23-25). The Kaplan-Meier plotter online (http://kmplot.com/analysis/) was used to predict the overall survival (OS) outcomes of patients with GC (26). This software contains a public database of Affymetrix microarray data from 1,065 patients with GC (ID, 211468_s_at). To analyze the prognostic value of RECQL5 in GC, the samples in the database were divided into 2 groups: High and low expression of RECQL5. The relationship between RECQL5 expression and survival data was analyzed using Kaplan-Meier survival curves. The log rank P-value and hazard ratio (HR) with $95 \%$ confidence intervals (CIs) were calculated.

GC tissue specimens and clinicopathological data. Informed consent was obtained from all individual participants included in the study, and the specimens were collected after approval from the Institute Research Medical Ethics Committee of The Sixth Affiliated Hospital, Sun Yat-sen University (Guangzhou, China). A total of 78 cancer specimens (age range, 38-76 years) were collected from patients with GC and matched with adjacent normal gastric tissues. The distance between tumor and normal tissues was $>1 \mathrm{~cm}$. The patients with GC underwent radical surgery between January 2009 and August 2011 at the Sixth Affiliated Hospital, Sun Yat-sen University (Guangzhou, China).

Immunohistochemistry. Paraffin embedded sections were used for immunohistochemistry. The thickness of the slides was $4 \mu \mathrm{m}$. Biotin-Streptavidin HRP Detection Systems (cat. no. SP-9001; Beijing Zhongshan Golden Bridge Biotechnology Co., Ltd.) was used to detect RECQL5 expression in GC samples. Staining was performed according to an established protocol using a rabbit polyclonal antibody against human RECQL5 (Sigma-Aldrich; Merck KGaA; cat no. HPA029971) diluted in PBS (1:150). Slides were incubated at $4^{\circ} \mathrm{C}$ in a moist chamber overnight with the primary antibody. Slides stained with PBS instead of primary antibody were used as negative controls. The visual immunoreactivity score (IRS) was calculated by using the following formula: Staining intensity (SI) $x$ percentage of stained cells with that intensity. IRS values were used to determine the expression level of RECQL5. The SI scores were as follows: 0 , negative; 1 , weak; 2 , moderate; and 3 , strong. The percentage of stained cells was calculated as the percentage of positively-stained tumor cells in the field, and was expressed as follows: 0, negative; 1, 0-25\%; 2, 26-50; $3,51-75 \%$; and $4,>76 \%$. Based on the SI scores, the RECQL5 expression level was classified as high (grades 4-12) or low (grades 0-3). Patients were classified into 2 groups, RECQL5 high and low. The tissues were independently scored by 2 pathologists who were blinded to the origin of each tissue. For any discrepancy, the 2 pathologists reassessed the slides together to reach an agreement.

Statistical analysis. SPSS version 22.0 (IBM Corp.) was used for statistical analyses. Ordinary one-way ANOVA was used to analyze the expression difference of RECQL5 from the Oncomine database. The association between clinicopathological features and RECQL5 protein expression was assessed using a $\chi^{2}$ test. The survival rate was assessed using Kaplan-Meier curves and the log-rank test. Cox proportional hazards regression model was applied for multivariate analysis to determine independent prognostic factors of GC. $\mathrm{P}<0.05$ was considered to indicate a statistically significant difference.

\section{Results}

RECQL5 $\mathrm{mRNA}$ and protein expression is low in patients with $G C$. Oncomine database analysis demonstrated that RECQL5 mRNA was downregulated in GC tissues compared with normal gastric tissues. The Cho Gastric dataset indicated that expression of RECQL5 was downregulated in diffuse gastric adenocarcinoma $(\mathrm{n}=31 ; \mathrm{P}=0.020)$, gastric adenocarcinoma $\left(\mathrm{n}=4 ; \mathrm{P}=6.82 \times 10^{-4}\right)$, and gastric mixed adenocarcinoma $(\mathrm{n}=10$; $\mathrm{P}=0.007)$ compared with that in normal gastric tissues $(n=19$; Fig. 1A-C). The Chen Gastric dataset revealed low expression of RECQL5 in diffuse gastric adenocarcinoma $(n=12$; $\left.\mathrm{P}=4.64 \times 10^{-8}\right)$, gastric intestinal type adenocarcinoma $(n=63$; $\left.\mathrm{P}=2.56 \times 10^{-4}\right)$, gastric mixed adenocarcinoma $\left(\mathrm{n}=8 ; \mathrm{P}=6.77 \times 10^{-5}\right)$ and compared with normal gastric tissues ( $n=26$; Fig. 1D-F). The DErrico Gastric dataset revealed that RECQL5 was downregulated in gastric mixed adenocarcinoma $(\mathrm{n}=4 ; \mathrm{P}=0.039)$, gastric intestinal type adenocarcinoma $(n=26 ; P=0.029)$ compared with normal gastric tissues ( $\mathrm{n}=31$; Fig. $1 \mathrm{G}$ and $\mathrm{H}$ ).

Immunohistochemistry was used to verify RECQL5 protein expression in GC and normal tissues. Expression of RECQL5 was found in GC tissues (Fig. 2D and E). Overall, 71.8\% (56/78 samples) of patients with GC displayed low RECQL5 expression in GC samples, while 28.2\% (22/78) displayed high RECQL5 expression. In the matched normal gastric tissues, $32.1 \%(25 / 78)$ of the patients displayed low RECQL5 expression and $67.9 \%(53 / 78)$ of the patients had high RECQL5 expression. The results indicated that RECQL5 expression was downregulated in GC tissues compared with normal gastric tissues $(\mathrm{P}<0.05$; Table I), consistent with the results of RECQL5 mRNA expression from the Oncomine database.

Association of RECQL5 differential expression and clinicopathological parameters of patients with GC. Low expression of RECQL5 was associated with depth of tumor invasion, histological differentiation and TNM stage $(\mathrm{P}<0.05)$, but not with patient age or sex, tumor size, lymph node metastasis, venous or lymphatic invasion or distant metastasis (P>0.05; Table II).

Low expression of RECQL5 predicts poor prognosis in patients with GC. The association between RECQL5 mRNA 
A

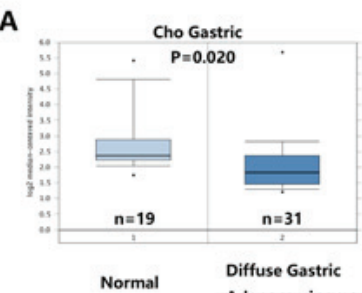

$\mathbf{E}$

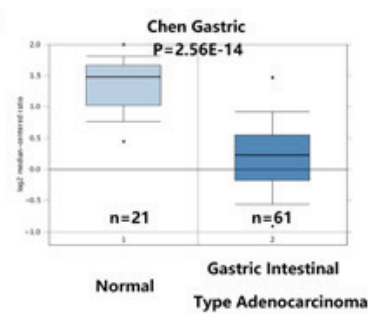

B

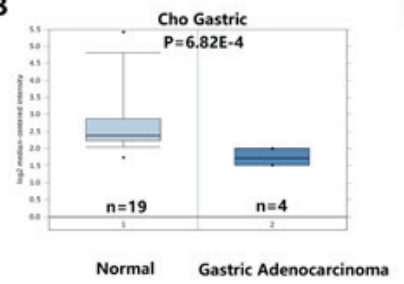

$\mathbf{F}$

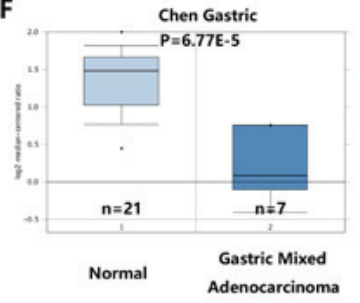

C

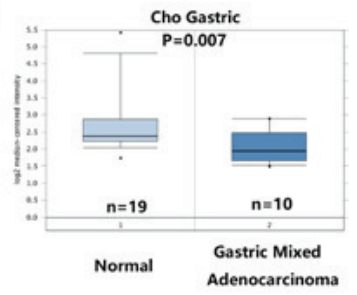

G

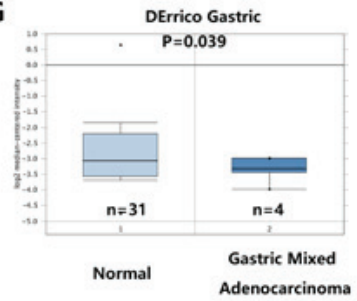

D

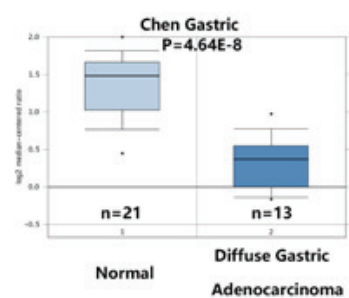

H

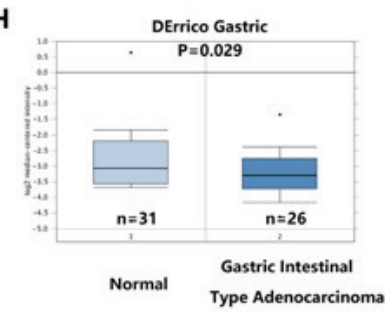

Figure 1. Expression of RECQL5 mRNA in human gastric cancer and normal gastric tissue using the Oncomine database. RECQL5 mRNA expression in (A-C) Cho Gastric dataset; (D-F) Chen Gastric dataset; (G and H) DErrico Gastric dataset. RECQL5, RecQ-like helicase 5.
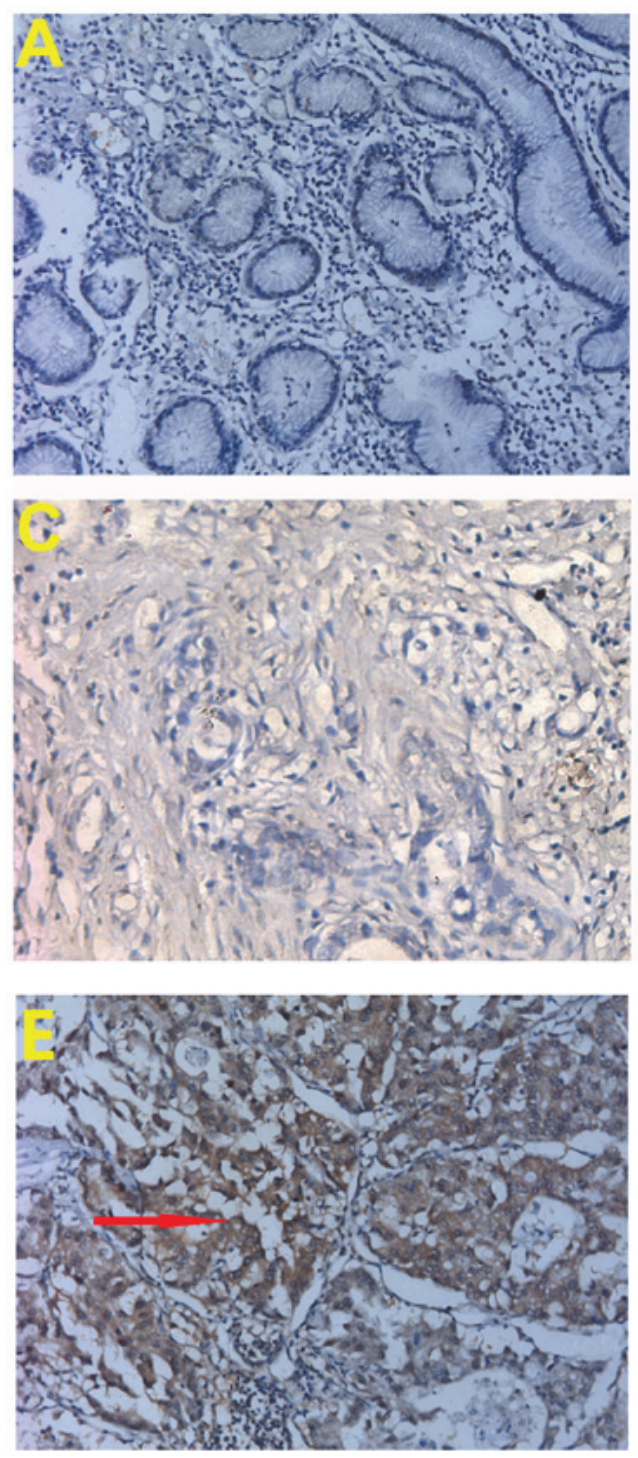
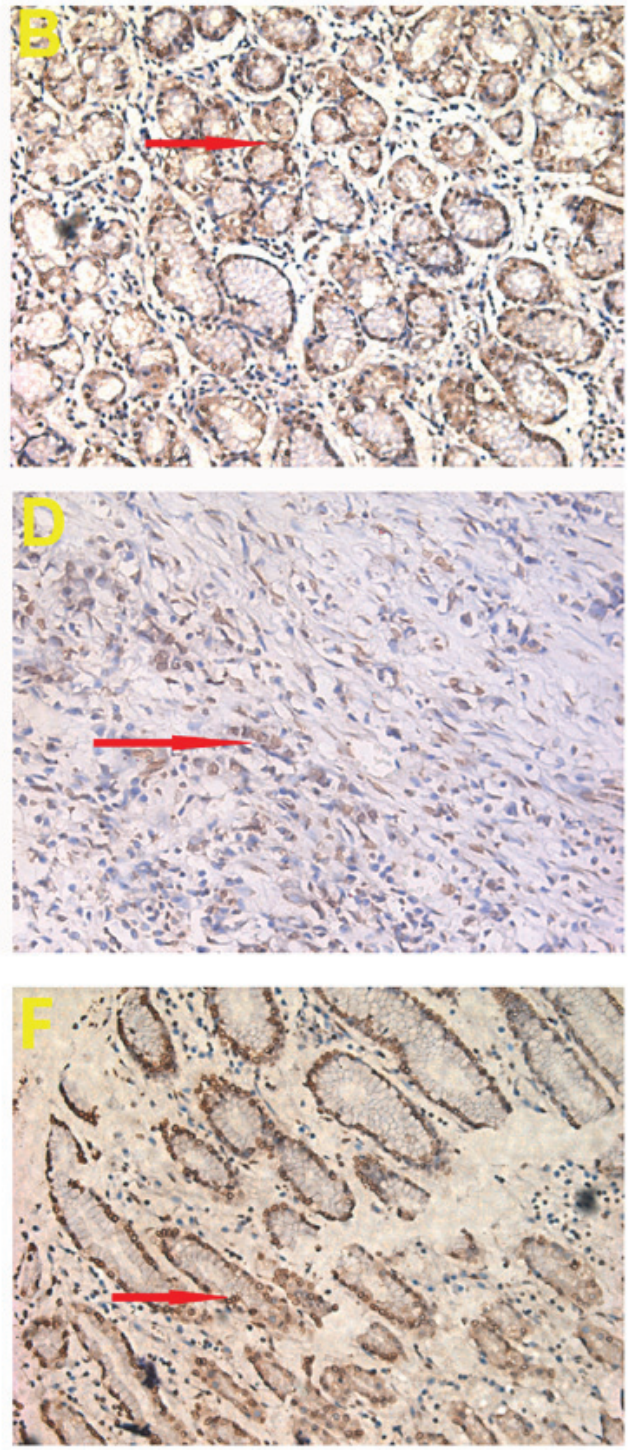

Figure 2. Immunohistochemical staining of RECQL5 in (A) normal gastric mucosal tissues with low expression of RECQL5; (B) normal gastric mucosal tissues with high expression of RECQL5; (C) GC samples with low expression of RECQL5; (D) GC samples with high expression of RECQL5 (poorly differentiated); (E) GC samples with high expression of RECQL5 (moderately differentiated); (F) GC samples with high expression of RECQL5 (well differentiated). The arrows represent RECQL5 protein expression. GC, gastric cancer; RECQL5, RecQ-like helicase 5. 
Table I. Expression of RECQL5 in normal gastric mucosa and primary gastric cancer tissues.

\begin{tabular}{lcccr}
\hline & & \multicolumn{2}{c}{ Expression of RECQL5 } & \\
\cline { 3 - 4 } Samples & Patients, $\mathrm{n}$ & Low, $\mathrm{n}(\%)$ & High, $\mathrm{n}(\%)$ & P-value \\
\hline Gastric cancer & 78 & $56(71.8)$ & $22(28.2)$ & $<0.001$ \\
Normal gastric tissue & 78 & $25(32.1)$ & $53(67.9)$ & \\
\hline
\end{tabular}

RECQL5, RecQ-like helicase 5.

Table II. Association between RECQL5 expression and clinicopathological features of patients with gastric carcinoma.

\begin{tabular}{|c|c|c|c|c|}
\hline \multirow[b]{2}{*}{ Characteristics } & \multirow[b]{2}{*}{ Patients, $\mathrm{n}$} & \multicolumn{2}{|c|}{ RECQL5 protein expression } & \multirow[b]{2}{*}{ P-value } \\
\hline & & Low, n (\%) & High, n (\%) & \\
\hline Sex & & & & 0.203 \\
\hline Male & 48 & $32(66.7)$ & $16(32.3)$ & \\
\hline Female & 30 & $24(80.0)$ & $6(20.0)$ & \\
\hline Age, years & & & & 0.240 \\
\hline$\geq 60$ & 45 & $30(66.7)$ & $15(33.3)$ & \\
\hline$<60$ & 33 & $26(78.8)$ & $7(21.2)$ & \\
\hline Tumor size, $\mathrm{cm}$ & & & & 0.094 \\
\hline$\geq 5$ & 31 & $19(61.3)$ & $12(38.7)$ & \\
\hline$<5$ & 47 & $37(78.7)$ & $10(21.3)$ & \\
\hline Histological differentiation & & & & 0.002 \\
\hline Well, moderate & 29 & $15(51.7)$ & $14(48.3)$ & \\
\hline Poorly, others & 49 & $41(83.7)$ & $8(16.3)$ & \\
\hline Depth of tumor invasion & & & & 0.019 \\
\hline $\mathrm{T} 1-\mathrm{T} 2$ & 30 & $17(56.7)$ & $13(43.3)$ & \\
\hline T3-T4 & 48 & $39(81.3)$ & $9(18.8)$ & \\
\hline Lymphatic invasion & & & & 0.957 \\
\hline Yes & 28 & $20(60.7)$ & $8(39.3)$ & \\
\hline No & 50 & $36(78.0)$ & $14(22.0)$ & \\
\hline Vascular invasion & & & & 0.881 \\
\hline Yes & 30 & $22(73.3)$ & $8(26.7)$ & \\
\hline No & 48 & $34(70.8)$ & $14(29.2)$ & \\
\hline Lymph node metastases & & & & 0.060 \\
\hline No & 33 & $20(60.6)$ & $13(39.4)$ & \\
\hline $\mathrm{N} 1, \mathrm{~N} 2$ & 45 & $36(80.0)$ & $9(20.0)$ & \\
\hline Distant metastasis & & & & 0.571 \\
\hline M0 & 53 & $37(69.8)$ & $16(30.2)$ & \\
\hline M1 & 25 & $19(76.0)$ & $6(24.0)$ & \\
\hline TNM stage & & & & 0.002 \\
\hline I-II & 32 & $17(53.1)$ & $15(46.9)$ & \\
\hline III-IV & 46 & $39(84.8)$ & $7(15.2)$ & \\
\hline
\end{tabular}

RECQL5, RecQ-like helicase 5; TNM, Tumor-Node-Metastasis.

expression levels and OS time in patients with GC was investigated using the Kaplan-Meier plotter software. Patients with a low expression of RECQL5 had a shorter OS time (HR, 0.84;
95\% CI,0.71-0.99; $\mathrm{P}=0.043$; Fig. 3A). The prognostic value of RECQL5 expression in GC was confirmed using the prognosis data of patients with GC from the Sixth Affiliated Hospital, 
Table III. Multivariate Cox regression analysis in patients with gastric cancer.

\begin{tabular}{|c|c|c|c|}
\hline Parameter & P-value & Hazard ratio & $95 \% \mathrm{CI}$ \\
\hline RECQL5, low vs. high & 0.002 & 2.922 & $1.504-5.679$ \\
\hline Histological differentiation, well, moderate vs. poorly, others & 0.326 & 1.533 & $0.653-3.598$ \\
\hline Depth of tumor invasion, T1-T2 vs. T3-T4 & 0.019 & 0.463 & $0.243-0.880$ \\
\hline TNM stage, I-II vs. III-IV & 0.544 & 0.772 & $0.336-1.778$ \\
\hline
\end{tabular}

CI, confidence interval; RECQL5, RecQ-like helicase 5.

A

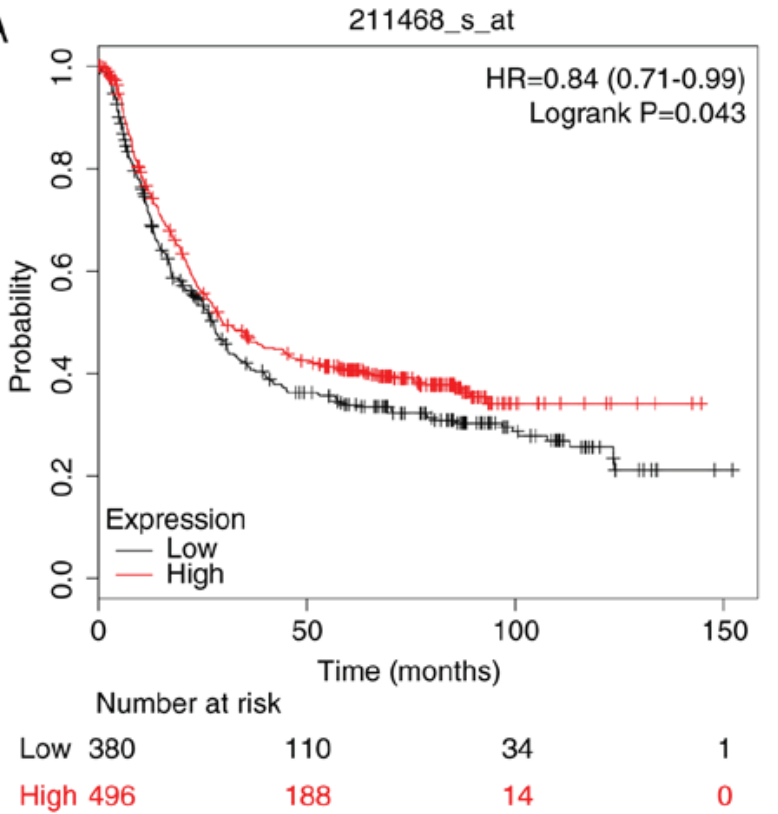

B

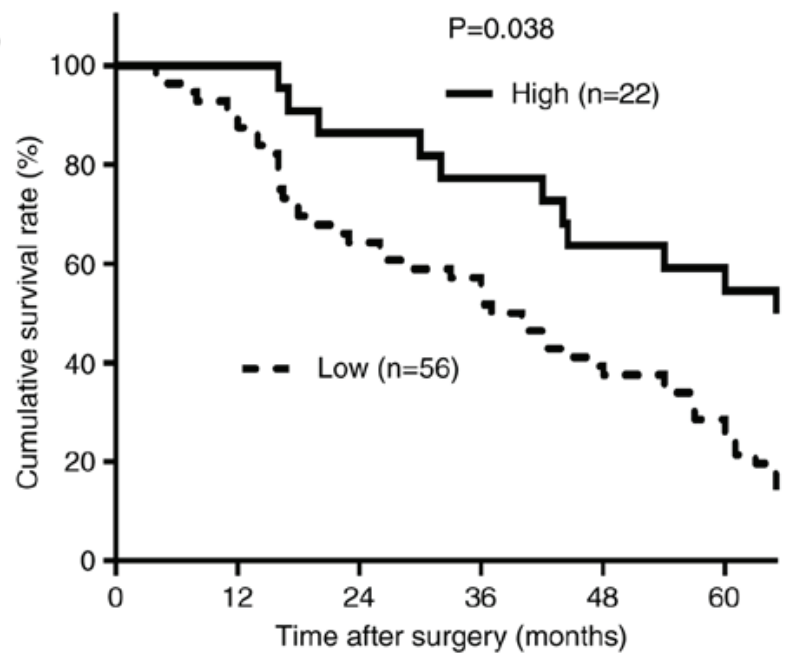

Figure 3. Association between RECQL5 expression levels and OS of patients with GC. (A) OS of patients with GC using the Kaplan-Meier plotter; (B) OS of patients with GC based on immunohistochemical staining. OS, overall survival; GC, gastric cancer; RECQL5, RecQ-like helicase 5.

Sun Yat-sen University (Guangzhou, China). The follow-up time ranged between 4 months and 9.5 years. The 5-year OS rate was $48.7 \%$ (38/78 patients). The 5-year OS rate of the RECQL5-low and RECQL5-high groups was 63.6\% (14/22) and $42.9 \%$ (24/56), respectively. Patients in the low RECQL5 expression group had a significantly shorter OS time $(\mathrm{P}=0.038$; Fig. 3B). The prognosis data was consistent with results from the Kaplan-Meier analysis. Multivariate analysis indicated that the independent prognostic factors were low expression of RECQL5 and depth of invasion $(\mathrm{P}<0.05$; Table III).

\section{Discussion}

Defects of WRN, BLM and RECQL4 may increase cancer predisposition in humans (10). However, whether RECQL5 is associated with cancer predisposition syndrome is unclear. Previous studies have shown that RECQL5 is an essential factor for maintenance of genomic stability, and that RECQL5 may act as an oncogene in various types of cancer $(20-22,27)$. Hu et al $(28,29)$ reported that RECQL5 regulates homologous recombination in mouse embryonic stem cells and downregulates the expression of RECQL5 in mice, which can increase susceptibility to colon carcinoma. Lao et al (19) demonstrated that loss of RECQL5 expression contributes to the pathogenesis of colorectal cancer. RECQL5 expression is also downregulated in osteosarcoma, and can inhibit proliferation and promote apoptosis of osteosarcoma cells (20). Conversely, a tumor-promoting function of RECQL5 was reported by several studies. Arora et al (21) reported the upregulation of RECQL5 in breast cancer due to gene amplification and described a critical role for RECQL5 in cancer progression and demonstrated that small interfering RNA-mediated knockdown of RECQL5 can significantly inhibit in vivo tumorigenicity and in vitro clonogenic survival of breast cancer cells (30). Patterson et al (22) identified a positive association between upregulated expression of RECQL5 with invasion of human urothelial bladder carcinoma. However, RECQL5 in patients with GC has not been fully investigated in previous studies.

In the present study, RECQL5 expression at the mRNA and proteins levels was significantly lower in GC tissues compared with normal gastric tissues. RECQL5 was expressed in 28.2\% of GC samples and $67.9 \%$ of matched normal gastric tissues. RECQL5 was localized mainly in the nucleus, similar to other studies $(19,20)$. In addition, the low expression of RECQL5 protein was associated with poor histological differentiation, 
deep tumor invasion and high tumor stage, indicating a prognostic role for RECQL5 in preventing GC progression. Furthermore, patients with high expression of RECQL5 had a higher 5-year OS rate compared with patients with low expression. Thus, low expression of RECQL5 might be a potential prognostic factor in GC. This was verified by multivariate analysis, which indicated that low expression of RECQL5 is an independent marker of poor prognosis, strengthening the hypothesis that RECQL5 may play an important role in preventing the progression of GC. The collective results of this study indicate that low expression of RECQL5 may be a predictor of poor prognosis in patients with GC.

RECQL5 is essential for maintaining genome stability and reducing cancer risk (28). RECQL5 has a tumor-suppressive role in the mouse gastrointestinal tract (29). The results of the present study indicate that the RECQL5 gene may be a candidate tumor suppressor gene in the stomach, and that high expression of RECQL5 may limit tumor growth. The present study also demonstrated that RECQL5 expression was high in normal gastric tissues, which may indicate that RECQL5 plays a role in maintaining genome stability and reducing cancer risk in the stomach. Moreover, low expression of RECQL5 may be a predictor of poor prognosis in patients with GC, which is consistent with previous reports $(29,30)$.

The present study has several limitations. This is a preliminary small-scale bioinformatics and clinical study. As the patient and normal samples were collected non-sequentially from a single center, a selection bias may exist in the study, which may have influenced the findings. Thus, further large-scale studies are required to validate the findings of the present study. In addition, in this study, only the expression of RECQL5 was investigated, and therefore, detailed studies to understand the molecular mechanisms of RECQL5 in GC are required. In conclusion, downregulation of RECQL5 was observed in GC samples. Low expression of RECQL5 was indicative of a more aggressive disease and might be an independent factor of poor prognosis in patients with GC.

\section{Acknowledgements}

Not applicable.

\section{Funding}

The present study was supported by the National Key Clinical Discipline, Guangdong Province Science and Technology Plan Project (grant no. 2017A010105004) and the Guangzhou Science and Technology Project (grant no. 201803010040).

\section{Availability of data and materials}

The datasets used and/or analyzed during the present study are available from the corresponding author on reasonable request.

\section{Authors' contributions}

YL, HC and JP designed the study. YL and HW analyzed and interpreted the patients' data and were the major contributors in writing the manuscript. HW analyzed and interpreted the patient data. XW and ML performed the histological experiments and were major contributors in writing the manuscript. All authors have read and approved the final version of the manuscript.

\section{Ethics approval and consent to participate}

The work was approved by the Institute Research Medical Ethics Committee of The Sixth Affiliated Hospital, Sun Yat-sen University (Guangzhou, China). This study has been performed in accordance with the Declaration of Helsinki. Informed consent was obtained from all individual participants included in the study.

\section{Patient consent for publication}

Not applicable.

\section{Competing interests}

The authors declare that they have no competing interests.

\section{References}

1. Balakrishnan M, George R, Sharma A and Graham DY: Changing trends in stomach cancer throughout the world. Curr Gastroenterol Rep 19: 36, 2017.

2. Chen W, Zheng R, Baade PD, Zhang S, Zeng H, Bray F, Jemal A, Yu XQ and He J: Cancer statistics in China, 2015. CA Cancer J Clin 66: 115-132, 2016.

3. Kankeu Fonkoua L and Yee NS: Molecular characterization of gastric carcinoma: Therapeutic implications for biomarkers and targets. Biomedicines 6: pii: E32, 2018.

4. Roberto M, Romiti A, Onesti CE,Zullo A,Falcone R and Marchetti P: Evolving treatments for advanced gastric cancer: Appraisal of the survival trend. Expert Rev Anticancer Ther 16: 717-729, 2016.

5. Imano $\mathrm{M}$ and Okuno $\mathrm{K}$ : Treatment strategies for gastric cancer patients with peritoneal metastasis. Surg Today 44: 399-404, 2014.

6. Yazici O, Sendur MA, Ozdemir N and Aksoy S: Targeted therapies in gastric cancer and future perspectives. World J Gastroenterol 22: 471-489, 2016.

7. Charalampakis N, Economopoulou P, Kotsantis I, Tolia M, Schizas D, Liakakos T, Elimova E, Ajani JA and Psyrri A: Medical management of gastric cancer: A 2017 update. Cancer Med 7: 123-133, 2018.

8. Arnold M, Moore SP, Hassler S, Ellison-Loschmann L, Forman D and Bray F: The burden of stomach cancer in indigenous populations: A systematic review and global assessment. Gut 63: 64-71, 2014.

9. Bernstein KA, Gangloff S and Rothstein R: The RecQ DNA helicases in DNA repair. Annu Rev Genet 44: 393-417, 2010.

10. Monnat RJ Jr: Human RECQ helicases: Roles in DNA metabolism, mutagenesis and cancer biology. Semin Cancer Biol 20: 329-339, 2010.

11. Rezazadeh S: RecQ helicases; at the crossroad of genome replication, repair, and recombination. Mol Biol Rep 39: 4527-4543, 2012.

12. Ouyang KJ, Woo LL and Ellis NA: Homologous recombination and maintenance of genome integrity: Cancer and aging through the prism of human RecQ helicases. Mech Ageing Dev 129: 425-440, 2008.

13. Qian X, Feng S, Xie D, Feng D, Jiang Y and Zhang X: RecQ helicase BLM regulates prostate cancer cell proliferation and apoptosis. Oncol Lett 14: 4206-4212, 2017.

14. Chun SG, Shaeffer DS and Bryant-Greenwood PK: The Werner's Syndrome RecQ helicase/exonuclease at the nexus of cancer and aging. Hawaii Med J 70: 52-55, 2011.

15. Frank B, Hoffmeister M, Klopp N, Illig T, Chang-Claude J and Brenner H: Colorectal cancer and polymorphisms in DNA repair genes WRN, RMI1 and BLM. Carcinogenesis 31: 442-445, 2010.

16. Zhu X, Chen H, Yang Y, Xu C, Zhou J, Zhou J and Chen Y: Distinct prognosis of mRNA expression of the five RecQ DNA-helicase family members-RECQL, BLM, WRN, RECQL4, and RECQL5-in patients with breast cancer. Cancer Manag Res 10: 6649-6668, 2018. 
17. Schwendener S, Raynard S, Paliwal S, Cheng A, Kanagaraj R, Shevelev I, Stark JM, Sung P and Janscak P: Physical interaction of RECQ5 helicase with RAD51 facilitates its anti-recombinase activity. J Biol Chem 285: 15739-15745, 2010.

18. Aygün O and Svejstrup JQ: RECQL5 helicase: Connections to DNA recombination and RNA polymerase II transcription. DNA Repair (Amst) 9: 345-353, 2010.

19. Lao VV, Welcsh P, Luo Y, Carter KT, Dzieciatkowski S, Dintzis S, Meza J, Sarvetnick NE, Monnat RJ Jr, Loeb LA and Grady WM: Altered RECQ helicase expression in sporadic primary colorectal cancers. Transl Oncol 6: 458-469, 2013.

20. Wu J, Zhi L, Dai X, Cai Q and Ma W: Decreased RECQL5 correlated with disease progression of osteosarcoma. Biochem Biophys Res Commun 467: 617-622, 2015.

21. Arora A, Abdel-Fatah TM, Agarwal D, Doherty R, Croteau DL, Moseley PM, Hameed K, Green A, Aleskandarany MA, Rakha EA, et al: Clinicopathological and prognostic significance of RECQL5 helicase expression in breast cancers. Carcinogenesis 37: 63-71, 2016.

22. Patterson K, Arya L, Bottomley S, Morgan S, Cox A, Catto J and Bryant HE: Altered RECQL5 expression in urothelial bladder carcinoma increases cellular proliferation and makes RECQL5 helicase activity a novel target for chemotherapy. Oncotarget 7: 76140-76150, 2016.

23. Chen X, Leung SY, Yuen ST, Chu KM, Ji J, Li R, Chan AS, Law S, Troyanskaya OG, Wong J, et al: Variation in gene expression patterns in human gastric cancers. Mol Biol Cell 14: 3208-3215, 2003.

24. D'Errico M, de Rinaldis E, Blasi MF, Viti V, Falchetti M, Calcagnile A, Sera F, Saieva C, Ottini L, Palli D, et al: Genome-wide expression profile of sporadic gastric cancers with microsatellite instability. Eur J Cancer 45: 461-469, 2009.
25. Cho JY, Lim JY, Cheong JH, Park YY, Yoon SL, Kim SM, Kim SB, Kim H, Hong SW, Park YN, et al: Gene expression signature-based prognostic risk score in gastric cancer. Clin Cancer Res 17: 1850-1857, 2011.

26. Szász AM, Lánczky A, Nagy Á, Förster S, Hark K, Green JE, Boussioutas A, Busuttil R, Szabó A and Győrffy B: Cross-validation of survival associated biomarkers in gastric cancer using transcriptomic data of 1,065 patients. Oncotarget 7: 49322-49333, 2016.

27. Dong YZ, Huang YX and Lu T: Single nucleotide polymorphism in the RECQL5 gene increased osteosarcoma susceptibility in a Chinese Han population. Genet Mol Res 14: 1899-1902, 2015.

28. Hu Y, Raynard S, Sehorn MG, Lu X, Bussen W, Zheng L, Stark JM, Barnes EL, Chi P, Janscak P, et al: RECQL5/Recq15 helicase regulates homologous recombination and suppresses tumor formation via disruption of Rad51 presynaptic filaments. Genes Dev 21: 3073-3084, 2007.

29. Hu Y, Lu X and Luo G: Effect of Recql5 deficiency on the intestinal tumor susceptibility of Apc(min) mice. World J Gastroenterol 16: 1482-1486, 2010.

30. He YJ, Qiao ZY, Gao B, Zhang XH and Wen YY: Association between RECQL5 genetic polymorphisms and susceptibility to breast cancer. Tumour Biol 35: 12201-12204, 2014 